\title{
Farber Lipogranulomatosis
}

National Cancer Institute

\section{Source}

National Cancer Institute. Farber Lipogranulomatosis. NCI Thesaurus. Code C84710.

A very rare autosomal recessive metabolic disorder affecting lipid metabolism. It is caused by mutations in the ASAH 1 gene and is characterized by fatty accumulation in the body tissues. Patients develop lipog ranulomas in the skin and internal organs, edema and pain in the joints and a hoarse voice. It may be associated with intellectual disability. 